\title{
Der Branklyn-Garten in Ost-Schottland
}

\author{
Hilke STEINecke
}

\section{Abstract}

IN 1922, Branklyn Garden was founded by Dorothy and John Renton. After they have deceased, te garden was taken over by the National Trust for Scotland. It is one of the most beautiful gardens of Scotland. It includes a fine collection of Himalayan and Chinese plants.

\section{Zusammenfassung}

Der Branklyn-Garten wurde 1922 von dem Ehepaar Renton angelegt. Nach Rentons Tod übernahm der National Trust for Scotland den Garten. Der kleine Garten gehört zu den schönsten Gärten Schottlands. Ein Sammlungsschwerpunkt sind Pflanzen aus dem Himalaya und China.

\section{Einführung}

Bei einer Schottlandreise im August denkt man zunächst wahrscheinlich an durch blühende Heide violett gefärbte Berge in den Highlands. In der Tat ist der Spätsommer eine besonders schöne Zeit, die Natur in diesem Fleckchen Nordwest-Europa zu erkunden. Außer der wilden Natur bieten sich aber auch einige Gärten zur Besichtigung an. Die attraktivsten Zeiträume sind vielleicht zwar eher der Frühling mit der Rhododendron-Blüte, der Frühsommer mit dem in fast allen Gärten üppig blühenden Blauen Scheinmohn (Meconopsis grandis) oder der Herbst mit einer intensiven Laubfärbung asiatischer Gehölze (z. B. Acer palmatum). Eigentlich gibt es aber immer etwas zu sehen. Wenn im Spätsommer in den Gärten die Farbe Grün dominiert, gewinnt der Besucher eine gute Vorstellung davon, wie das mild-feuchte, regenreiche atlantische Klima für das Wachstum verschiedenster Gewächse geeignet ist. Selbst ein verregneter Tag, wie die Autorin ihn im

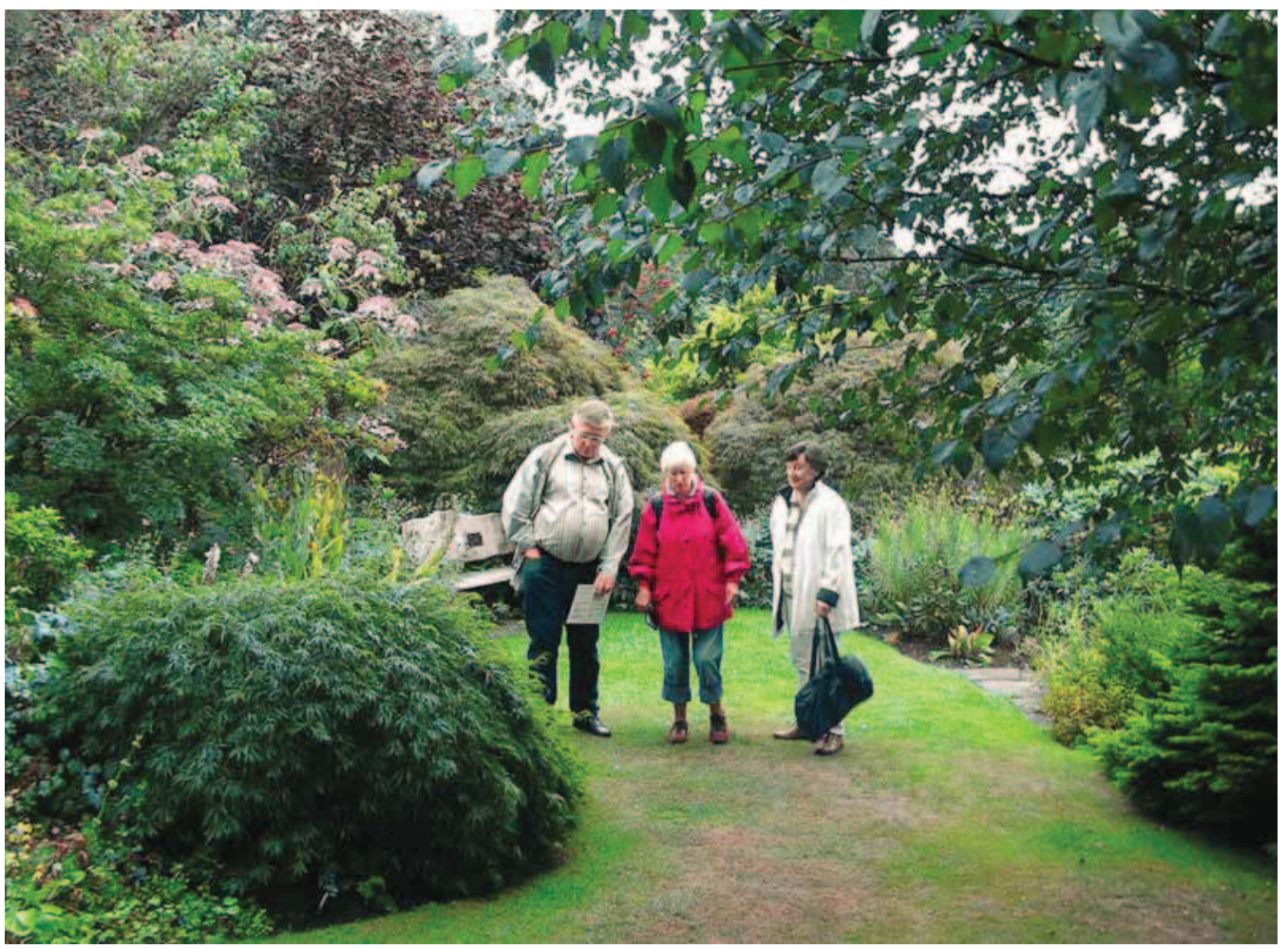



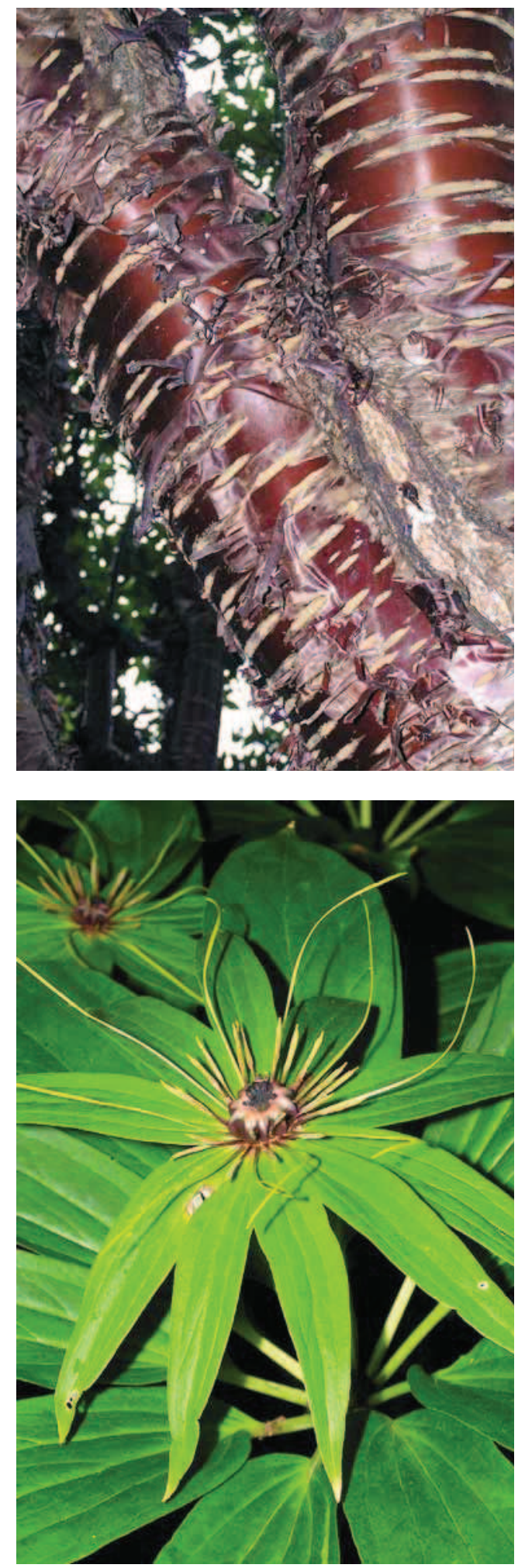

Branklyn-Garten erlebte, hat seine Reize, wenn Wassertropfen einzelne Blüten zieren und die nassen Blätter schwer an den Zweigen hängen.

\section{Der Garten und seine Geschichte}

Der Branklyn-Garten befindet sich im Osten Schottlands in der Nähe von Perth im BarnhillBezirk nahe des Flusses Tay. Das Gelände liegt mit 30-40 m Höhe nur unwesentlich über dem Meeresspiegel. Der Garten wurde 1922 von Dorothy und John Renton angelegt. Die Eheleute hatten kurz zuvor das Grundstück, einen Teil eines alten Obstgartens, erworben. Sie bauten sich ein Wohnhaus, das zunächst nur von einem kleinen Garten umgeben war. Doch bald wurde die Gartenarbeit zu einer Passion der Eheleute. Sie erwarben innerhalb weniger Jahre zusätzlichen Grund, ebenfalls Stücke eines alten Obstgartens, um den Garten zu erweitern. Insgesamt ist der Garten heute 0,7 ha groß.

$\mathrm{Zu}$ Beginn der Planung und Anlage des Gartens wurden zwar einige Fehler gemacht, wie Dorothy Renton behauptete, aber man lernte dazu, so dass der Garten nach und nach immer schöner wurde. Eine der ersten Anlagen war der Steingarten. Mit Hilfe einer Dampfmaschine wurden schwere Steine aus der Umgebung herangeschafft. Die meiste Arbeit wurde jedoch von den Rentons und ihrem Gärtner in mühseliger Handarbeit erledigt.

In den 1940er Jahren erhielten sie zusätzliche Hilfe von James Aitken, dem Sohn des ehemaligen Obstgartenbesitzers. Es war den Rentons ein wichtiges Anliegen, den einzelnen Pflanzen optimale Wachstumsbedingungen zu bieten, was einerseits die Arbeit erleichtert und anderseits die Pflanzen besonders üppig wachsen lässt. Die Rentons vermuteten, dass in dem mild-feuchten Klima Schottlands und auf dem sauren Boden ihres Grundstückes mit einem pH-Wert zwischen 4,8 und 6,1 Pflanzen aus

Abb. 1 (Seite 25): Auf grasbewachsenen Pfaden im Garten. Abb. 2 (oben): Der rote Stamm von Prunus serrula. Abb. 3 (unten): Daiswa polyphylla ist mit der Einbeere verwandt. 
dem asiatischen Raum (China, Himalaya) sehr gut gedeihen würden. Sie knüpften deshalb Kontakte zu Pflanzenjägern und Expeditionsleitern und erhielten schließlich 200 Päckchen mit Samen, die an Wildstandorten in SüdostTibet und Bhutan gesammelt worden waren. Rhododendren und Hortensien sind an vielen Stellen des Gartens anzutreffen.

Ihre gestalterischen Ideen wurden von GeRTRUDE JeKYll beeinflusst. Die Rentons legten großen Wert auf farbliche Harmonie der Pflanzungen, wobei nicht nur die Farbe der Blüten, sondern auch der Blätter bei der Gestaltung berücksichtigt wurden. Noch heute zeugen in der Nähe des Hauses Ahorne mit roten oder goldgelben Blättern, die mit den dunklen Nadeln von Koniferen kontrastieren, von diesen Ideen. Hervorhebenswert ist eine Kiefer mit kugelförmiger Krone (Pinus sylvestrisf. globosa). Diese Form ist in Kultur meist als kleine Konifere bekannt, während dieser über 70 Jahre alte Baum mehrere Meter hoch ist. Ein Charakteristikum des hinteren Gartenteils sind die kleinen Terrassen und die Torfmauern. Hier wachsen Moorbeetpflanzen, z.B. aus den Gattungen Vaccinium, Gaultheria, Kalmia und Phyllodoce.

Der Garten entwickelte sich in relativ kurzer Zeit aufgrund seiner Sammlung seltener und attraktiver Arten zu einem Vorzeigeobjekt. John Renton war für planerische Aufgaben zuständig. DоRотнY, die eigentliche Gärtnerin in der Anlage, erhielt für ihre Arbeit verschiedene Auszeichnungen: 1954 die Vеiтch-Medaille der Royal Horticultural Society und 1960 die Scottish Horticultural Medal. Vom National Trust for Scotland wurde bereits zu ihren Lebzeiten das Sammeln und Austesten ausgefallener Pflanzen unterstützt. DоRотнY und JoHn starben 1966 bzw. 1967. Danach gingen Haus und Garten in den Besitz des National Trust for Scotland über. Bis heute ist es ein Anliegen, den

Abb. 4 (oben): Raoulia haastii.

Abb. 5 (Mitte): Aus der Ferne wirken die Blüten von Cyananthus wie Enziane.

Abb. 6 (unten): Garten-Shop.
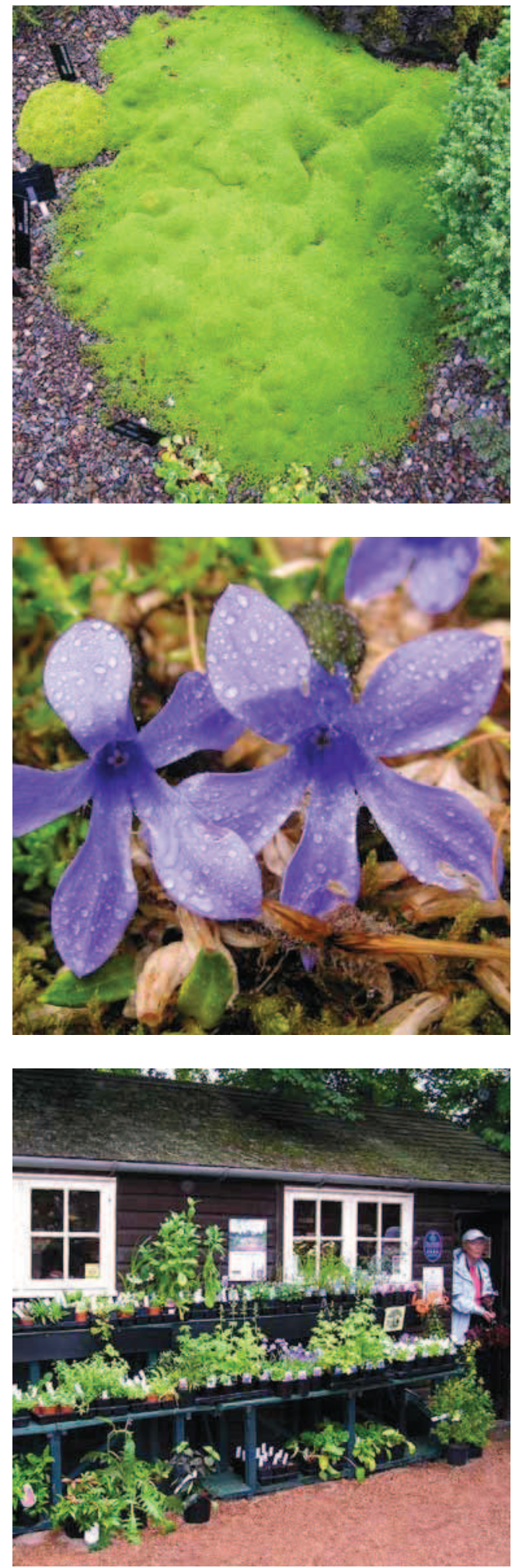

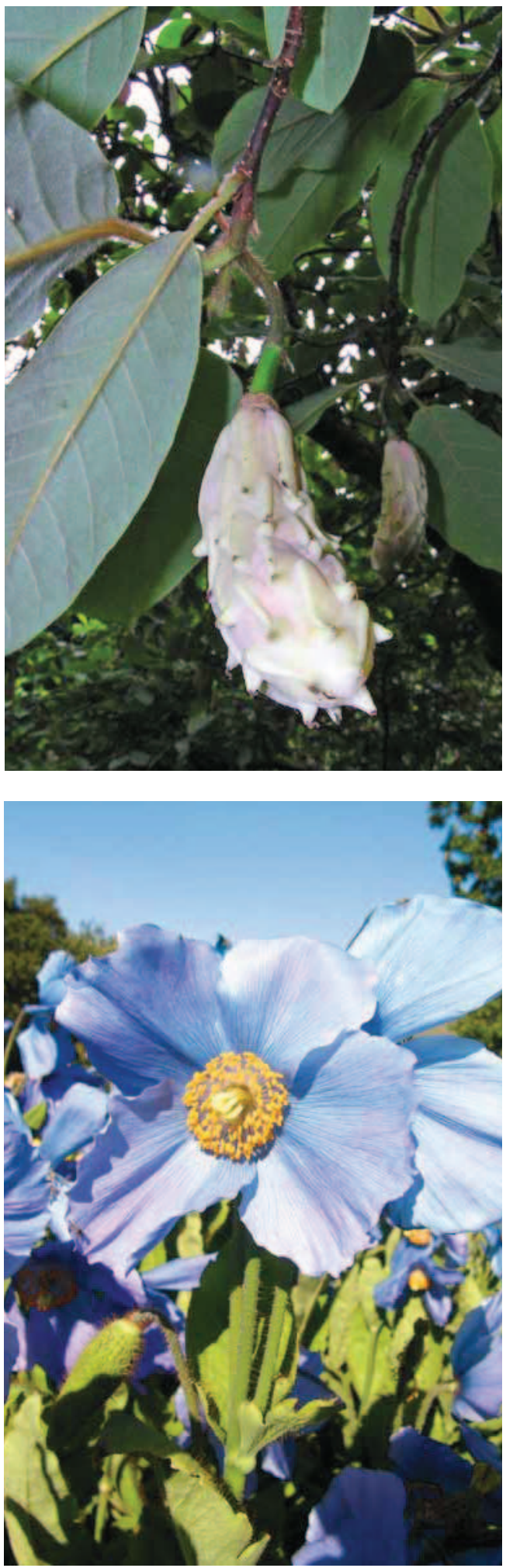

Garten im Sinne seiner Begründer weiterzuführen. An vielen Ecken sind die Spuren ihrer gärtnerischen Tätigkeiten noch zu sehen.

\section{Ein kleiner Rundgang durch den Garten} Man kann den Garten auf zwei schmalen, verwunschenen Wegen erkunden. Da die Blicke kleinräumig auf Details fallen, wirkt der Garten größer, als er eigentlich ist. In der Nähe des Hauses befindet sich ein kleiner Teich, an dessen Ufer Gunnera sehr gut gedeiht. Blickfang sind im Spätsommer vor allem Gehölze mit einer auffällig gefärbten Borke. Besonders markant sind der rotbraun glänzende Stamm von Prunus serrula oder die rötlich bis rosa gefärbte Rinde von Betula albo-sinensis var. septentrionalis. Diese Birke wurde aus Samen gezogen, die JosepH Rock von seiner Expedition nach SüdwestChina mitgebracht hatte. Der Wiener JosEPH Rock (1884-1962) gilt als einer der angesehensten Erforscher der Flora von China und Hawaii. Zudem studierte er die Kultur und Sprache der Menschen in China (vor allem in Yunnan, Sichuan, Gansu) sowie in Ost-Tibet. Von 1922 bis 1949 unternahm er mehrere Forschungsreisen nach China. Er sammelte dabei tausende Pflanzen, Samenproben und Herbarbelege. Auf den Pflanzenjäger ERnest Wilson weist Magnolia wilsonii, die ca. 1932 gepflanzt wurde, hin. WILsON (1876-1930) war besonders in China als Pflanzensammler unterwegs, weshalb er auch den Spitznamen Chinese WILson erhielt. Vor allem im Auftrag der Gärtnerei VEITCH führte er viele interessante Zierpflanzen nach England ein. Zimt-Ahorn (Acer griseum), Taschentuchbaum (Davidia involucrata) oder Hartriegel (Cornus kousa var. chinensis) wurden von WILSON zwischen 1900 und 1907 nach Europa gebracht. Diese Gehölze findet man heute in vielen schottischen Gärten, auch im Branklyn-Garten. Nur selten wird in Gärten an der Ostküste Schottlands Trochodendron ara-

Abb. 7 (oben): Magnolia wilsonii wurde nach dem Pflanzenjäger ERNEST WiLson benannt.

Abb. 8 (unten): Im Sommer blühen in Schottlands Gärten verschiedene Meconopsis-Arten. 
lioides aus Japan kultiviert, im Branklyn-Garten ist er völlig winterhart. Im August bildet Eucryphia glutinosa zahlreiche, etwa sechs $\mathrm{cm}$ breite, weiße Blüten mit zahlreichen Staubblättern. Dieser kleine Baum aus Chile ist dann eines der auffälligsten Blütengehölze im Garten. Ein alter Birnbaum ist Zeuge davon, dass das Gelände einst als Obstgarten genutzt wurde.

Zwischen den Gehölzen gedeihen verschiedene Stauden, von denen der Blaue Mohn (Meconopsis betonicifolia, M. x sheldonii, M. horridula, M. grandis) besonders attraktiv ist. Da der heiße und trockene Sommer 2006 auch in Schottland seine Spuren hinterlassen hat, waren viele Sommerblumen, so auch der Blaue Mohn, leider bereits verblüht. Im Sommer gedeihen im Garten auch Lilium superbum, Hosta, verschiedene Enziane oder Cardiocrinum giganteum. Im Garten ist eine Nationalsammlung von Cassiope und Lilium angesiedelt. Dem Garten ist die Pfingstrosen-Sorte Paeonia 'Branklyn' gewidmet.

Der Steingarten beherbergt besonders Arten aus Nepal und China. Eine Rarität ist die mit dem Seidelbast verwandte Stellera chamaejasme, von der es in Großbritannien nur noch zwei Exemplare gibt, die ursprünglich von DoNALD Lowndes 1950 aus dem Nepal eingeführt wurden. Es ist geplant, den Garten auch in Zukunft mit Pflanzen aus Nepal und Yunnan zu bereichern. Eine kissenförmig wachsende Hebe cupressoides 'Boughton Dome' steht seit der Einrichtung des Steingartens an ihrem Platz. Im kalten Winter und Frühjahr 2005/2006 erlitt sie Frostschäden. Das äußerst feste Polster der aus Neuseeland stammenden Raoulia haastii ist so robust, dass man darauf sitzen könnte. Bereits Dorothy Renton ließ sich gern darauf nieder.

Wer einen detaillierteren Überblick über den Artenbestand des Gartens bekommen möchte, sollte sich den im Internet veröffentlichten Index Seminum ansehen. Zudem gibt es einen Gartenführer. In einem kleinen, liebevoll eingerichteten Shop kann der Besucher kleine Töpfe mit

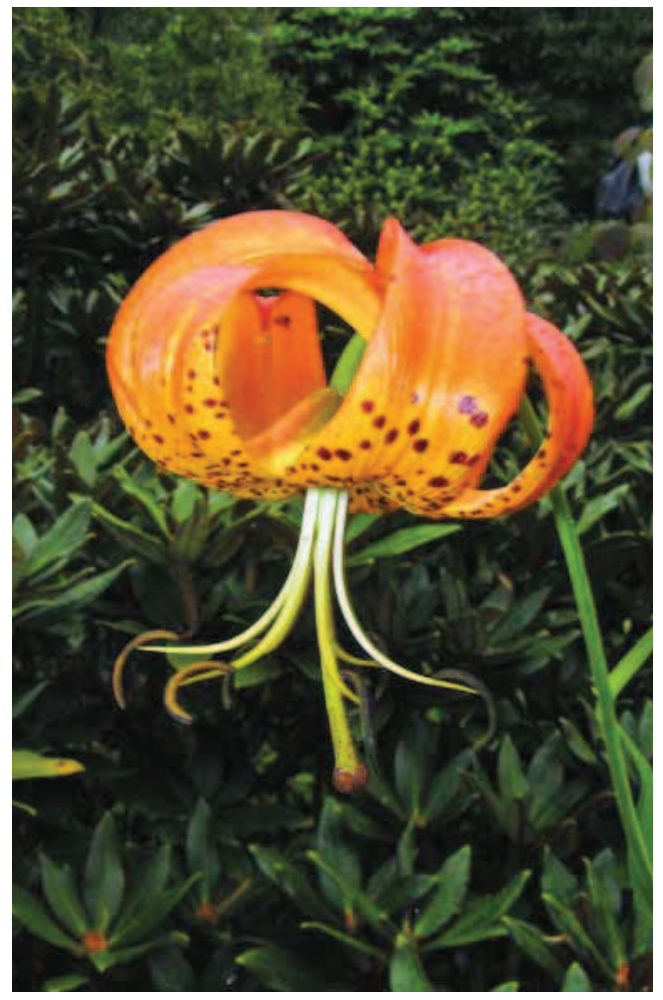

Stauden erwerben. Der Garten ist für die Öffentlichkeit vom 1. April bis 31. Oktober von 10.00 Uhr-17.00 Uhr geöffnet.

\section{Literatur}

Mitchell, B. 1992: Branklyn Garden. - Edinburgh (eine Neuauflage ist in Vorbereitung).

\section{Internetseiten}

http://www.wildview.me.uk/branklyn/history.htm www.branklyngarden.org.uk/ 